\title{
Clinical Spectrum of Adrenal Cushing's Syndrome and the Caution for Interpretation of Adrenocorticotrophic Hormone: A Single- Center Experience
}

Authors

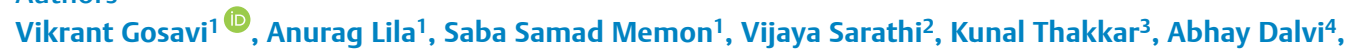
Gaurav Malhotra ${ }^{5}$, Gagan Prakash ${ }^{6}$, Virendra Patil'1, Nalini S. Shah¹, Tushar Bandgar ${ }^{1}$

Affiliations

1 Department of Endocrinology, Seth G. S. Medical College and KEM Hospital, Mumbai, India

2 Department of Endocrinology, Vydehi Institute of Medical Sciences and Research Centre, Bangalore, India

3 Sterling Ramkrishna Speciality Hospital, Gandhidham, India

4 Department of Surgery, Seth G. S. Medical College and KEM Hospital, Mumbai, India

5 Department of Nuclear Medicine, Bhabha Atomic Research Centre, Mumbai, India

6 Department of Uro-oncology, Tata Memorial Hospital, Mumbai, India

Key words adrenal Cushing's syndrome, adrenocorticotrophic hormone, Cushing's syndrome, adrenocortical carcinoma

received 31.08 .2021

accepted after revision $\quad 03.01 .2022$

\section{Bibliography}

Horm Metab Res 2022; 54: 57-66

DOI 10.1055/a-1735-3232

ISSN 0018-5043

(C) 2022. Thieme. All rights reserved. Georg Thieme Verlag, Rüdigerstraße 14,

70469 Stuttgart, Germany

Correspondence

Dr. Anurag Lila

Department of Endocrinology

103, 1st Floor

OPD Building

KEM Hospital Campus

Parel

400012 Mumbai

Maharashtra

India

Tel.: + 91/800/7774 385

anuraglila@gmail.com

\section{ABSTRACT}

To describe the differences in presentation, biochemistry, and radiological evaluation of various etiologies of adrenal Cushing's syndrome (CS) from a single center. To emphasize caution for interpretation of plasma adrenocorticotropic hormone (ACTH), as a spuriously unsuppressed ACTH level by immunometric assay may lead to therapeutic misadventures in adrenal CS. Design: Retrospective, single-center, observational study. Methods: Fifty-eight adrenal CS patients [Adrenocortical carcinoma (ACC), $n=30$; Adenoma (ACA), $n=15$; Primary pigmented nodular adrenocortical disease (PPNAD), $n=10$; ACTH independent macronodular adrenal hyperplasia (AIMAH), $n=3$ ) evaluated at a tertiary care center in western India between January 2006 to March 2020 were included. Data on demography, clinical evaluation, biochemistry, imaging, management, histopathology, and outcome were recorded in a standard format and analyzed. Results: Cortisol secreting ACC presented at $38(1-50)$ years with abdominal mass in $26 / 30$ (86.7\%) and $16 / 30$ (53.3\%) had metastases at presentation. ACA with autonomous cortisol excess presented at 25(4.9-40) years with discriminating features of CS in 14/15 (93.3\%), sex steroid production in $2 / 15$, unenhanced $\mathrm{HU}<10$ in only one, and relative washout $>40 \%$ in $8 / 11$ (72.7\%). One ACA and eight ACC patients had plasma ACTH (by Siemens Immulite assay) $>20 \mathrm{pg} / \mathrm{ml}$, despite hypercortisolemic state. Conclusions: Cortisol-secreting ACC and ACA most often present with mass effects and florid CS, respectively. Baseline HU has low sensitivity to differentiate cortisol-secreting ACA from ACC. Plasma ACTH measured by Seimens Immulite is often unsuppressed, especially in ACC patients, which can be addressed by measuring ACTH by more accurate assays. 


\section{Introduction}

Endogenous Cushing's syndrome (CS) is characterized by excess and unregulated cortisol secretion leading to adverse clinical outcomes. Hypersecretion of cortisol may be driven either by an excessive adrenocorticotrophic hormone (ACTH) from the pituitary/ ectopic source or by a primary adrenal pathology - adrenal CS. Adrenal CS is a less frequent cause of CS, accounting for $20 \%$ of adult patients, whereas it is more common in children (50\% in children $<7$ years of age) [1, 2]. Unilateral pathology (tumor) is predominant, whereas bilateral pathology (hyperplasia) accounts for a smaller proportion of patients. Adrenocortical carcinoma (ACC) has a bimodal age distribution (first and 5-6th decade), whereas adrenocortical adenoma (ACA) is more prevalent in the 4-5th decade [3]. Bilateral causes also have age predilection with primary pigmented nodular adrenocortical disease (PPNAD) manifesting in the first three decades and primary bilateral macronodular adrenal hyperplasia (PBMAH) presenting in the 5-6th decade [4]. Understanding the differences in presentation, biochemistry, and radiological evaluation of adrenal CS is vital for their appropriate management.

Measurement of plasma ACTH level by immunometric assay helps in the etiological classification of endogenous CS. Plasma ACTH level $>20 \mathrm{pg} / \mathrm{ml}$ indicates ACTH-dependent CS, while $\leq 10 \mathrm{pg} / \mathrm{ml}$ indicates ACTH-independent (adrenal) etiology. ACTH levels $>10-20 \mathrm{pg} / \mathrm{ml}$ fall in the 'grey zone', and an additional battery of tests is warranted for distinguishing ACTH-dependent from ACTH-independent etiologies [5, 6]. Inappropriate sample collection and storage conditions may lead to falsely low ACTH levels due to degradation of this labile analyte, which may erroneously suggest an ACTH-independent CS. On the other hand, adrenal CS may be misdiagnosed as ACTH-dependent by an unsuppressed plasma ACTH, as reported in a few case reports/series [7-10]. Here, we describe our experience of adrenal CS from a single center, emphasizing caution for interpretation of ACTH.

\section{Ethics Approval}

The study was approved by the Institutional Ethics Committee-II (EC/OA-101/2019) of Seth GS Medical College and KEM hospital with a waiver of consent.

\section{Patients and Methods}

A retrospective data analysis of patients with adrenal CS, diagnosed and managed at our institute between January 2006 and March 2020, a tertiary referral health care center in western India, was done. The final etiological diagnosis of adrenal CS was based on histopathology in all, except for five metastatic ACC and two PBMAH patients in whom the diagnosis was based on clinical, hormonal, and imaging characteristics. In adrenocortical tumor patients with available histopathology (surgical specimen in 36 cases and biopsy in 4 cases), the presence of metastasis, local invasion or recurrence, and/or a Weiss score $\geq 4$, were used to diagnose ACC whereas in those without metastasis, local invasion or recurrence and Weiss score of $\leq 3$, a diagnosis of ACA were made. Data on demography, clinical evaluation, biochemistry, imaging, management, histopathology, and outcomes were recorded in a standard format. In a patient presenting with adrenal mass, autonomous cortisol se- cretion was diagnosed by an overnight dexamethasone suppression test (ODST) serum cortisol value was $>5 \mu \mathrm{g} / \mathrm{dl}$ and/or 24 -hour urinary free cortisol (UFC) was more than the upper limit of normal (ULN). Patients with possible autonomous cortisol secretion (ODST cortisol 1.9-5.0 $\mu \mathrm{g} / \mathrm{dl}$ ) were excluded. Moon facies with plethora, easy bruising, wide livid striae, proximal myopathy, and weight gain with reduced growth velocity in children were considered as discriminatory signs of CS (DSCS) [11]. In clinically suspected CS with biochemically proven endogenous hypercortisolism and suppressed plasma $A C T H \leq 10 \mathrm{pg} / \mathrm{ml}$ were subjected to adrenal imaging. Plasma ACTH level was repeated in patients with borderline plasma ACTH (10-20 pg/ml). Contrast-enhanced CT adrenal imaging was performed with a 64-slice multidetector CT system (Brilliance 64, Philips Healthcare, Best, and The Netherlands), and absolute and relative washout characteristics of adrenal masses were noted as per standard protocol [12]. ${ }^{18} \mathrm{~F}$-Fluorodeoxyglucose positron emission tomography-computed tomography (FDG-PET/CT) was performed as per standard protocol for patients with suspected malignancy. The highest standardized uptake value (SUVmax) was determined by software incorporated in the PET workstation. It was defined as a focal area of abnormal uptake in the region of interest (ROI) compared to the surrounding.

Patients with ACA underwent laparoscopic adrenalectomy, while adrenal tumors suspicious of ACC underwent open surgical resection. Patients with unilateral lesions who had post-surgery 8:00 AM serum cortisol level $<5 \mu \mathrm{g} / \mathrm{dl}$ were diagnosed to have suppressed hypothalamic-pituitary-adrenal (HPA) axis and were replaced with oral glucocorticoid. Patients with uncured/inoperable ACC were managed with local bed radiotherapy and/or chemotherapy regimen with etoposide, doxorubicin, and cisplatin with or without mitotane.

Cortisol was measured by a solid-phase competitive chemiluminescent enzyme immunoassay (Siemens Healthcare) with an analytical sensitivity of $0.2 \mu \mathrm{g} / \mathrm{dl}$. This assay's intra-assay and interassay coefficients of variability (CV) were 6.9 and $7.3 \%$, respectively. Plasma ACTH was measured on a solid-phase, two-site sequential chemiluminescent assay. Immulite (Siemens Healthcare) assay has been used since 2006, and Liaison (Diasorin) was added since December 2017. The intra-assay, interassay CV, and analytical sensitivity were $9.6 \%, 8.8 \%, 0.5 \mathrm{pg} / \mathrm{ml}$, and $4.9 \%, 8.9 \%, 1.6 \mathrm{pg} / \mathrm{ml}$ for Siemens Immulite and Liaison assays, respectively. The lowest plasma ACTH value was considered for analysis when multiple values were available. Plasma ACTH was measured with adequate pre-analytic care (collection of plasma sample, maintaining a cold temperature, and immediate processing). Serum DHEAS was measured by Chemiluminescence Microparticle Immunoassay (CMIA) on Rosch Cobas platform with intra-assay and inter-assay (CVs) of 4 and $4.6 \%$, respectively, and analytical sensitivity of $0.2 \mu \mathrm{g} / \mathrm{dl}$. We have used the age and gender-specific normative range for serum DHEAS as described previously $[13,14]$.

\section{Statistical analysis}

Qualitative data were represented as frequency and percentage. Quantitative data were described using mean \pm standard deviation for normally distributed data, otherwise as median with range. Association between qualitative variables was assessed by the chi-square test or Fisher's exact test. Analysis of quantitative data 
between two groups was carried out using unpaired $t$-test or Mann-Whitney test. For all statistical tests, $p<0.05$ was considered significant. Receiver operating characteristic (ROC) curve analysis was performed to differentiate ACC from ACA using tumor size and relative washout. Statistical analysis was performed using IBM SPSS Statistics (version 23.0) and MedCalc for Windows (version 19.8).

\section{Results}

Of the total endogenous CS patients $(n=310), 18.7 \%(n=58)$ had adrenal CS. The characteristics of the study cohort are described in - Table 1. Unilateral adrenal pathology was more common [ACC $=30(51.7 \%), A C A=15(25.9 \%)]$ compared to bilateral causes $[P P N A D=10(17.2 \%), P B M A H=3(5.2 \%)]$. Patients with PPNAD were the youngest [19 (1.2-48) years], whereas those with PBMAH were the oldest [57 (30-60) years]. There was female preponderance, which was most marked in the ACA group. DSCS was more frequent in ACA (93.3\%) and PPNAD (100\%) than ACC (46.7\%) and PBMAH (1/3 patient).

On comparing ACC with ACA, ACC primarily presented as abdominal mass (86.7\%) while most ACA presented with CS (80\%). ACA presented at a younger age and had female predilection. Basal and ODST serum cortisol were comparable between the two groups. Plasma ACTH levels were significantly higher (15.9 \pm 13.3 vs. $8.8 \pm 12.0 \mathrm{pg} / \mathrm{ml})$ in the ACC group than in the ACA group. Twelve patients $(A C C=10, A C A=2)$ had plasma $A C T H$ levels between 10 and $20 \mathrm{pg} / \mathrm{ml}$ and nine patients $(A C C=8, A C A=1)$ had levels $>20 \mathrm{pg} / \mathrm{ml}$.

Low serum DHEAS had a sensitivity of $54.5 \%$ (6/11) and specificity of $83.3 \%(15 / 18)$ for the diagnosis of ACA. Elevated serum DHEAS had a sensitivity of $50 \%(9 / 18)$ and specificity of $81.8 \%$ (9/11) for the diagnosis of ACC. A patient of ACA (5-year-old girl) had presented with abdominal mass (size: $5.6 \mathrm{~cm}$ ) and also had pubarche and clitoromegaly with a serum testosterone level of $1.1 \mathrm{ng} / \mathrm{ml}$ and DHEAS level of $185.3 \mu \mathrm{g} / \mathrm{dl}$ (normal range: 7.4-46.8). Another 13-year-old boy with the final diagnosis of ACA (size: $5.2 \mathrm{~cm}$ ) had presented with CS; also had gynecomastia with a serum estrogen level of $246 \mathrm{pg} / \mathrm{ml}$ and DHEAS level of $367 \mu \mathrm{g} / \mathrm{dl}$ (normal range: 13.6-288.9). Both these patients had a Weiss score of 3 on histopathological examination. Adrenal tumor size $>4 \mathrm{~cm}$ had a sensitivity of $96.7 \%(29 / 30)$ and specificity of $73.3 \%(11 / 15)$ for the diagnosis of ACC. This specificity increased to $100 \%$, with a sensitivity of $93.3 \%(28 / 30)$ when tumor size cut off was increased to $>6 \mathrm{~cm}$. Basal HU was $>10$, for all adrenal tumors, except one ACA $(1 / 11$, $9.1 \%)$. Absolute washout of $>60$ and $\geq 55 \%$ had sensitivities of $63.6 \%(7 / 11)$ and $90.9 \%(10 / 11)$ and specificities of $72.2 \%(13 / 18)$ and $66.7 \%(12 / 18)$, respectively, for the diagnosis of ACA. Relative washout of $>40 \%$ had a sensitivity of $72.7 \%(8 / 11)$ and specificity of $100 \%$ (18/18) for the diagnosis of ACA. On FDG-PET/CT, the lesion SUVmax of ACC was significantly higher than that of ACA $(12.9 \pm 4.8$ vs. $6.2 \pm 1.8, p=0.019)$, and the ratio of lesion SUVmax to liver SUVmean was $6.5 \pm 2.6(n=10)$. In the ACA cohort, lesion SUVmax was $6.2 \pm 1.8(n=3)$, and lesion SUVmax to liver SUVmean ratio was $1.9(n=1)$. Using ROC curve analysis, a lesion size of more than of $5.4 \mathrm{~cm}$ had sensitivity and specificity of 93.3 and $99.93 \%$ respectively, for the diagnosis of ACC whereas a relative washout of more than $31.8 \%$ had sensitivity and specificity of 90.9 and $95 \%$, respectively, for the diagnosis of ACA.

The plasma ACTH values for adrenal CS patients are depicted in - Fig. 1. One ACA and eight ACC patients had plasma ACTH of $>20 \mathrm{pg} / \mathrm{ml}$ despite hypercortisolemic state as defined by ODST serum cortisol value $>5 \mu \mathrm{g} / \mathrm{dl}$ and/or UFC $>$ ULN ( $>$ Table 2). Repeat ACTH values were not available for these ACC patients as they had presented with abdominal pain leading to detection of large adrenal mass $(8.9-19 \mathrm{~cm})$ on ultrasonogram. Of these eight ACC patients, only three had DSCS. While the ACA patient with ACTH above $20 \mathrm{pg} / \mathrm{ml}$ was a 21-year-old female, presented with CS and an unsuppressed plasma ACTH $(49.6 \mathrm{pg} / \mathrm{ml})$. Her pituitary imaging was normal, and inferior petrosal sinus sampling was planned. Meanwhile, contrast-enhanced CT chest and abdomen was done to localize an obvious ectopic source, which revealed a $3.2 \mathrm{~cm} \mathrm{li-}$ pid-poor (unenhanced CT density: $27 \mathrm{HU}$ ) left adrenal mass with relative washout of $47.1 \%$, with a thinned out right adrenal. She was cured after the left adrenalectomy. ACTH values in these patients were obtained from the Seimens Immulite platform. In another five patients with initial ACTH of $>13 \mathrm{pg} / \mathrm{ml}(13.1-149 \mathrm{pg} /$ $\mathrm{ml})(13.1-149 \mathrm{pg} / \mathrm{ml})$ by Siemens Immulite assay, repeat ACTH measurements by Liaison assay were $<13 \mathrm{pg} / \mathrm{ml}(1.6-12.7 \mathrm{pg} / \mathrm{ml})$.

Most ACC patients (53.3\%) were of ENSAT (European Network for the Study of Adrenal Tumors) stage IV, while stage II and III comprised $20 \%$ patients each, and only two patients presented with stage I disease. Surgery was not considered feasible in nine out of the 16 metastatic patients, of which 5 received palliative chemotherapy. Twenty-one patients underwent open surgery followed by local bed radiotherapy $(n=9)$ and chemotherapy $(n=13)$. The median survival of the ACC group was 23 ( $95 \% \mathrm{Cl}: 3$ to 43 ) months ( Fig. 2). All ACA patients were cured following laparoscopic excision of the tumor. The mean time to HPA axis recovery post-surgery was $10.6 \pm 9.1$ months.

Most (9/10) of the PPNAD patients have been described previously [15]. Two patients of AIMAH presented incidentally on abdominal imaging, while one presented with CS. The latter, who underwent bilateral adrenalectomy, were cured, while the other two were managed with watchful observation (annual monitoring with 24-hour UFC) and medical therapy for comorbidities (diabetes mellitus and hypertension).

\section{Discussion}

In our cohort, adrenal CS comprised $18.7 \%$ of all endogenous CS, with unilateral etiology (77.6\%) being more common than bilateral (22.4\%). We report that plasma ACTH level of $>20 \mathrm{pg} / \mathrm{ml}$ is not uncommon in adrenal CS when measured by Siemens Immulite assay, especially in patients with ACC. All ACC with an ACTH of $>20 \mathrm{pg} / \mathrm{ml}$ were detected by ultrasonogram. Washout characteristics (relative washout $<40 \%$, absolute washout of $<60 \%)$ and size $(>5.4 \mathrm{~cm})$, but not baseline $\mathrm{HU}$, had good diagnostic accuracy to differentiate cortisol- secreting ACC from ACA.

The proportion of adrenal CS amongst endogenous CS is similar to that reported in the literature $(\approx 20 \%)[1,2,6]$. In contrast, amongst the adrenal CS, the proportion of ACC (despite being a predominant adult cohort) was more than ACA, which may be due to referral bias. 

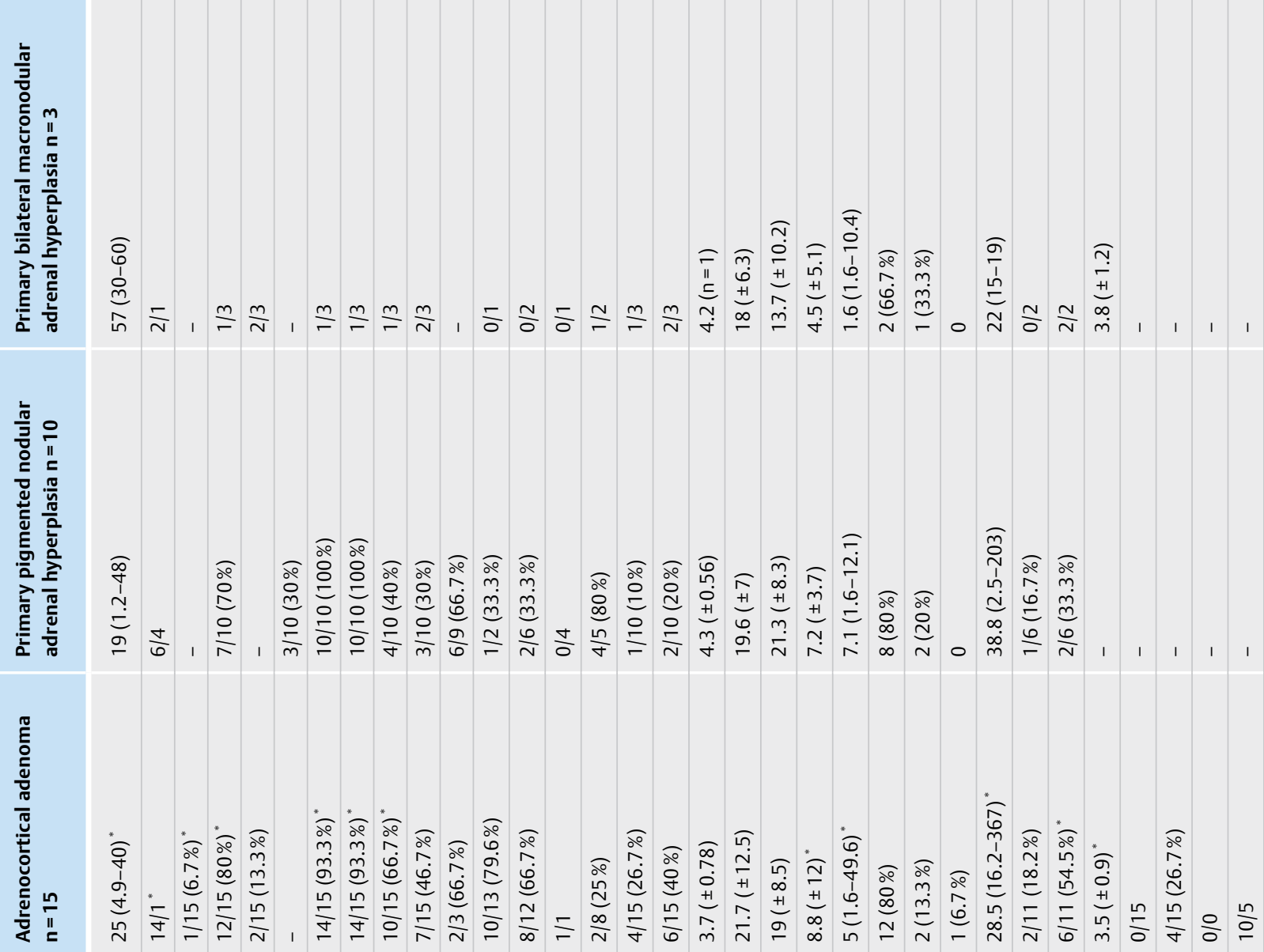

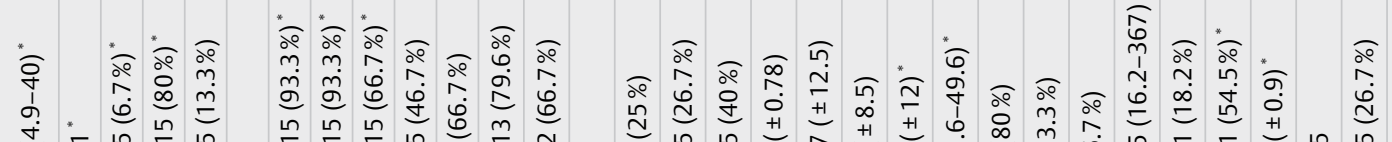

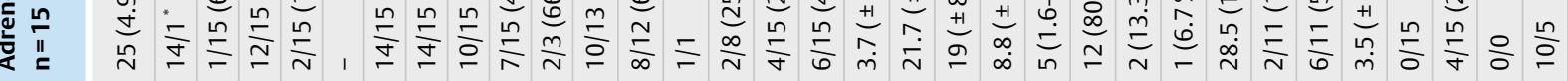

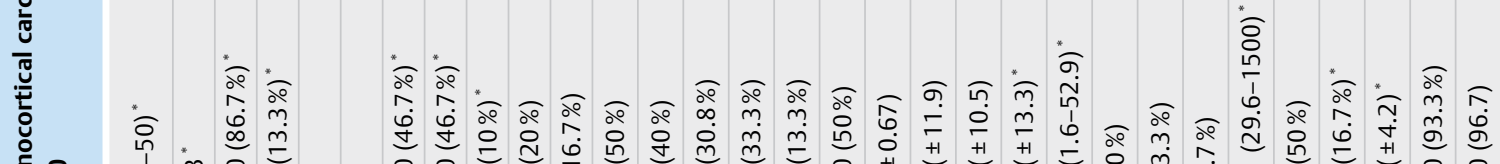

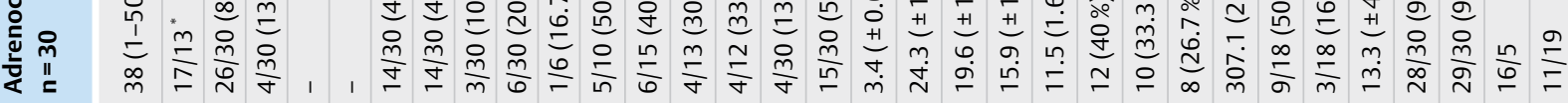

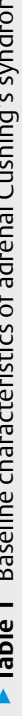
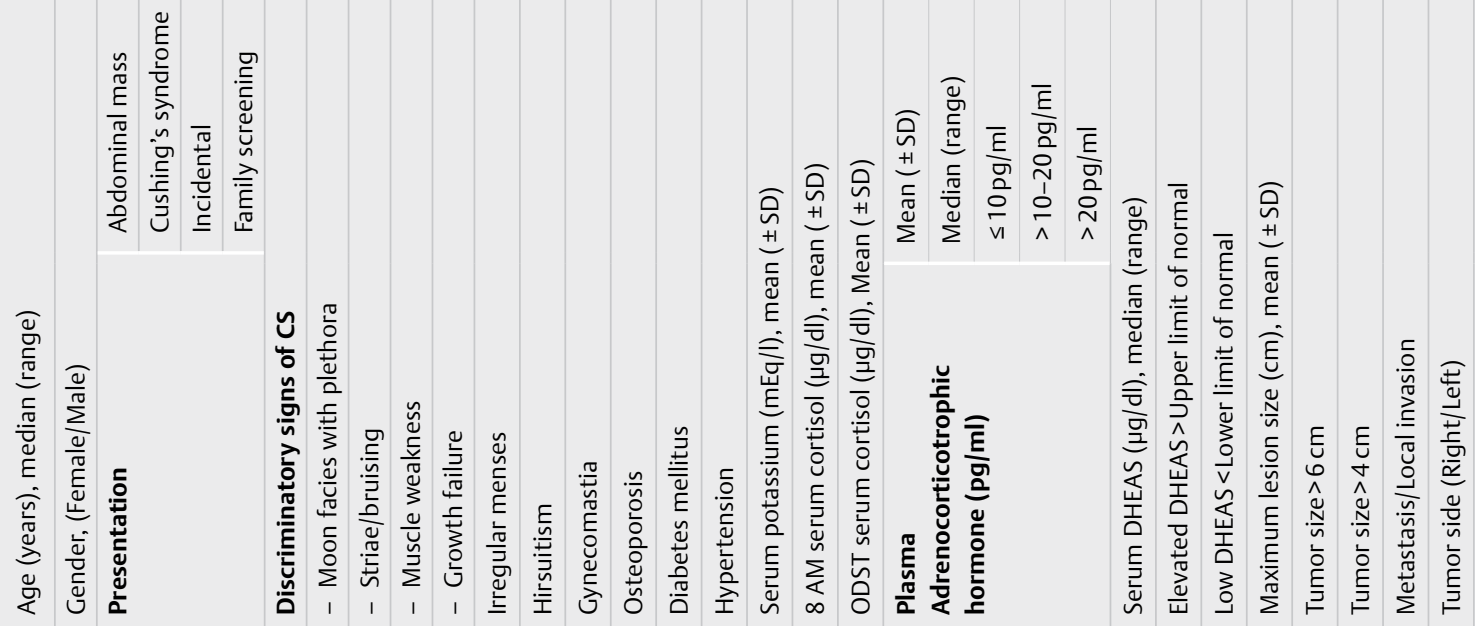


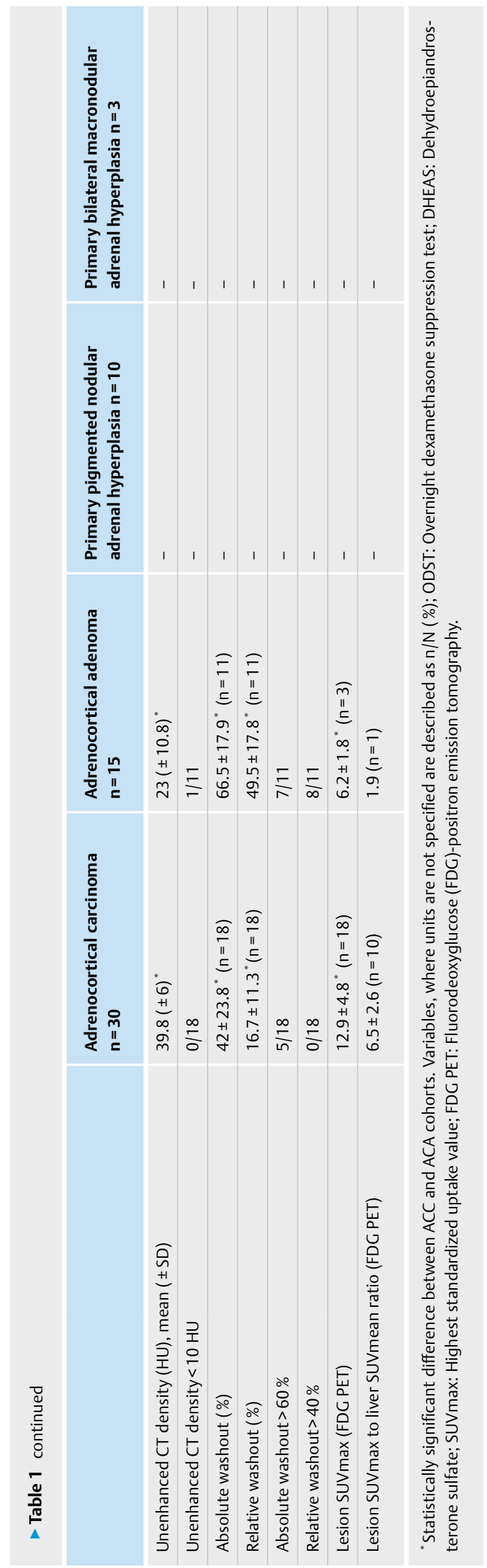

The younger age of our ACC cohort may represent the earlier age at presentation of cortisol-secreting ones, as also reported in a previous Indian study [16], than unselected ACC cohorts. Despite cortisol hypersecretion in all, clinical features of CS were present in only $46.7 \%$ of patients. This observation suggests considering a biochemical evaluation for CS in all ACC patients, irrespective of the clinical features. The majority (86.7\%) of patients in our cohort had presented with abdominal symptoms, possibly due to delay in seeking healthcare or inefficient cortisol secretion or action (masked by the anabolic action of androgens). In addition, 53.3\% of patients had distant metastases at presentation, compared to lower metastasis rates ( 25 to $47.4 \%$ ) in larger studies $[3,17]$. This could reflect either delayed diagnosis or more aggressive nature of the disease in cortisol-secreting ACC. Corresponding with the presentation, the median survival in our cohort was also shorter than reported cohorts $[17,18]$.

The ACA subgroup in our study had a mean age of $25.7 \pm 10.5$ years, and predominant presentation due to overt CS in $80 \%$ of patients, which could be due to selection bias as patients with possible autonomous cortisol production (ODS cortisol 1.8 to $5 \mu \mathrm{g} / \mathrm{dl}$ ) were not included in the cohort. In addition, we had two pediatric patients who presented with co-secretion of sex steroids. Co-secretion of cortisol with sex steroids by ACA is rarely described and should raise the suspicion for malignancy $[3,19,20]$. Both of these patients had a Weiss score of 3 on histopathological examination and were disease-free at the last follow-up ( 5 and 5.5 years). However, low-grade ACC cannot be ruled out in both these patients and are under close surveillance for recurrence of the disease. The mean duration for recovery of the HPA axis after surgery in our cohort was similar to the reported 11.2 months in literature [21].

In general, $71 \%$ of benign adrenal masses have a basal $\mathrm{HU}$ of $<10$ (lipid-rich) [22]. In contrast, only $9.1 \%$ of cortisol secreting adenoma were lipid-rich in our study. Similar observations were made in two studies from France, one reporting $12 \%$ (3/25) and another reporting $20.6 \%$ (7/34) of cortisol-secreting ACA having a basal HU of $<10[23,24]$. In a clinicopathological correlation study, unlike aldosterone-secreting ACA that were characterized by lipid-rich clear cells, most of cortisol-secreting ACA were characterized by the presence of granule cells [25]. Similarly, in another study from France, lipid-poor (baseline attenuation: > $10 \mathrm{HU}$ ) cortisol-secreting ACA had $<25 \%$ lipid-rich clear cells in $50 \%$ [24]. In this study, the magnitude of cortisol hypersecretion in cortisol-secreting ACA was associated with lipid-poor nature, both on histopathology ( $<25 \%$ clear cells) and radiology (baseline HU). Hyperfunctioning nature with depletion of intracytoplasmic lipid droplets containing cholesterol esters necessary for cortisol synthesis or upregulation of cAMP pathway with a change from lipid-rich clear cell to lipid-poor compact cell have been hypothesized to account for the lipid poor nature of cortisol-secreting ACA. Hence, basal attenuation of $<10 \mathrm{HU}$ has less diagnostic sensitivity for cortisol secreting ACA. However, a baseline attenuation of $<10 \mathrm{HU}$ excludes ACC, irrespective of secretory status, and can be useful to differentiate ACA, including a small proportion of cortisol-secreting ones, with this radiological characteristic (10-20\%) from ACC [23, 24, 26].

Size of the tumor $>6 \mathrm{~cm}$ was $100 \%$ specific for the diagnosis of ACC, but sensitivity was $93.3 \%$ (28/30). Poor relative contrast washout ( $<40 \%$ ) was $100 \%$ sensitive for the diagnosis of ACC, but spec- 


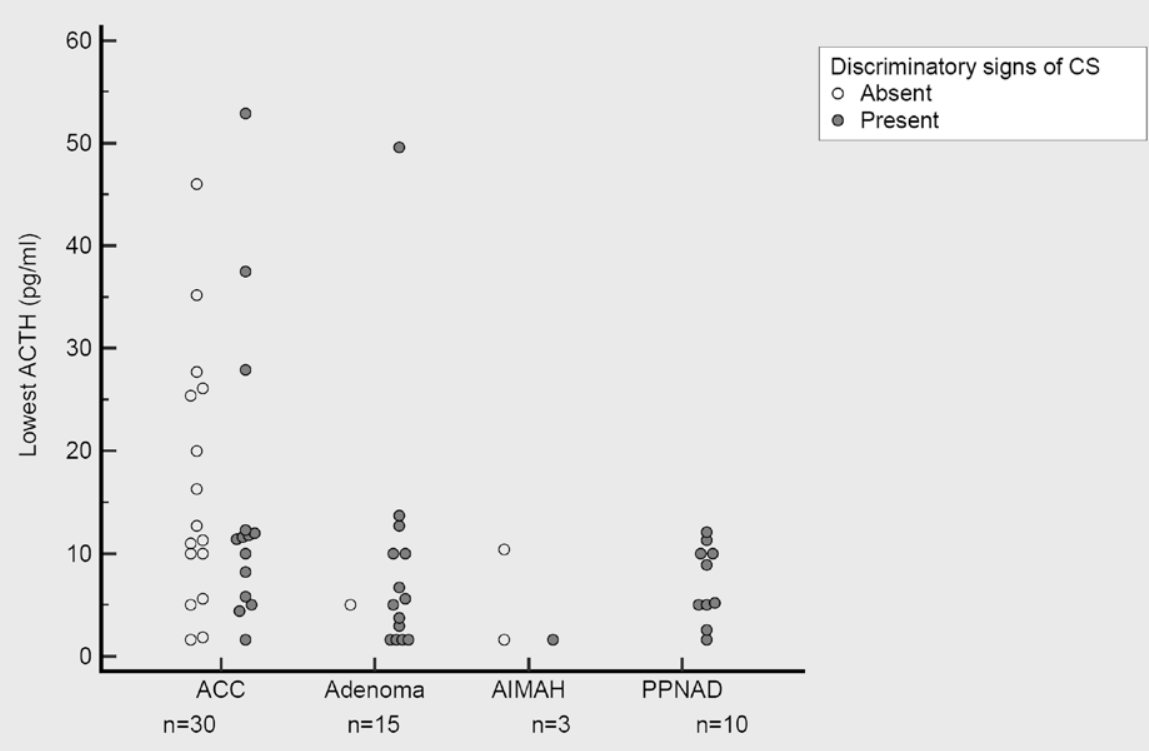

- Fig. 1 Scatter diagram of adrenocorticotrophic hormone (ACTH) levels in the total study cohort $(n=58)$.

- Table 2 Adrenal Cushing syndrome (CS) patients with plasma adrenocorticotrophic hormone (ACTH)>20 pg/ml from this study.

\begin{tabular}{|c|c|c|c|c|c|c|c|c|}
\hline $\begin{array}{l}\text { Patient } \\
\text { No }\end{array}$ & Age/sex & Presentation & $\begin{array}{l}\text { Discriminatory } \\
\text { signs of CS }\end{array}$ & $\begin{array}{l}8 \text { AM serum } \\
\text { cortisol }(\mu \mathrm{g} / \mathrm{dl})\end{array}$ & $\begin{array}{l}\text { ODST Serum } \\
\text { Cortisol ( } \mu \mathrm{g} / \mathrm{dl})\end{array}$ & $\begin{array}{l}\text { Plasma ACTH pg/ml } \\
\text { (Assay platform) }\end{array}$ & $\begin{array}{l}\text { Tumor size } \\
(\mathrm{cm})\end{array}$ & $\begin{array}{l}\text { Final } \\
\text { diagnosis }\end{array}$ \\
\hline P 1 & $45 / F$ & Abdominal mass & No & 20.3 & 7 & $35.2(\mathrm{SI})$ & 12 & ACC \\
\hline P 2 & $40 / F$ & Abdominal mass & No & 17.9 & 17.8 & 26.1 (SI) & 11.6 & ACC \\
\hline P 3 & $46 / M$ & Abdominal mass & No & 11.3 & 11.8 & $25.4(\mathrm{SI})$ & 10.6 & ACC \\
\hline P 4 & $30 / M$ & Abdominal mass & No & 10.8 & 8.4 & $46(\mathrm{SI})$ & 19 & ACC \\
\hline P 5 & $45 / M$ & Abdominal mass & No & - & 9 & $27.7(\mathrm{SI})$ & 18.9 & ACC \\
\hline P6 6 & $45 / F$ & Abdominal mass & Yes & 19.3 & 25.1 & $27.9,(\mathrm{SI})$ & 13 & ACC \\
\hline P 7 & $29 / F$ & Abdominal mass & Yes & - & $-\mathrm{a}$ & $52.9(\mathrm{SI})$ & 10 & ACC \\
\hline P 8 & $42 / M$ & Abdominal mass & Yes & 32.8 & $--^{a}$ & 37.5 (SI) & 8.9 & ACC \\
\hline P9 & $21 / \mathrm{F}$ & Cushing's syndrome & Yes & 28.5 & 28 & 49.6 (SI) & 3.2 & ACA \\
\hline
\end{tabular}

a Urinary free cortisol was 1.7 and 7.3 times the upper limit of normal for P7 and P8, respectively; F: Female, M: Male; ACC: Adrenocortical carcinoma; ACA: Adrenocortical adenoma; ODST: Overnight dexamethasone suppression test; SI: Siemens Immulite.

ificity was $72.7 \%$, as few [3/11 (27.3\%)] ACA exhibited poor washout. This is in concordance with data in general, where lipid-poor adenoma has poor washout in $41 \%$ of the patients [27]. Hence, a role FDG-PET/CT has been suggested by He et al. to differentiate the malignant lesions (higher SUVmax and higher lesion SUVmax to liver SUVmean ratio) from benign ones [28]. Similar values of these two parameters were noted in our study too. Notably, a patient with ACC had a lesion size of $<6 \mathrm{~cm}$, but the lesion SUVmax of 11.6 was suggestive of malignancy. Similarly, a patient of ACA with poor washout characteristics had a lesion SUVmax of 6.7, which was indicative of its benign nature. Also, higher FDG uptake has been observed in cortisol-secreting ACA in comparison to non-secreting ones [23].
PBMAH patients of our study presented later in life either with subclinical $(n=2)$ or clinical CS $(n=1)$, which is similar to the available literature. Unlike ACC, ACTH was suppressed in all three patients despite subclinical CS in two patients. The decision for bilateral adrenalectomy or medical management was individualized based on the clinical severity of hypercortisolemia and comorbidities, as suggested previously [4].

We report that plasma ACTH of $>20 \mathrm{pg} / \mathrm{ml}$ is not uncommon in patients with adrenal CS, especially in ACC. In contrast, unsuppressed ACTH in adrenal CS is limited to a few cases in the literature. Adrenal CS cases reported in the literature (ODST serum cortisol value $>5 \mu \mathrm{g} / \mathrm{dl}$ and/or 24-hour UFC more than the upper limit of normal) with unsuppressed ACTH are summarized in > Table 3. Unsuppressed ACTH in adrenal CS may prompt unnecessary inves- 


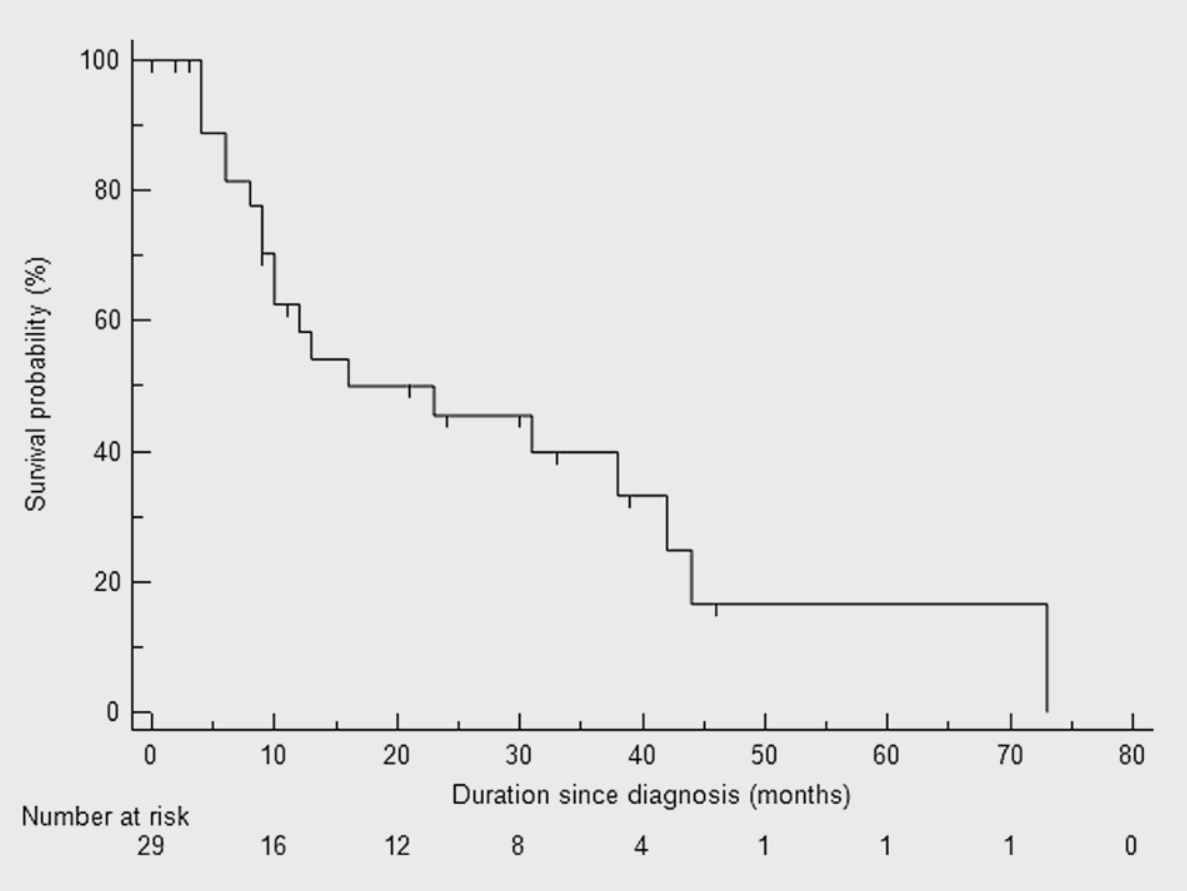

- Fig. 2 Kaplan-Meier survival curve of the total cohort of adrenocortical carcinoma $(n=30)$.

tigations like MRI pituitary, IPSS, CT thorax and neck, and/or nuclear scans, and in unfortunate instances, even an unnecessary pituitary surgery [7-9]. Unsuppressed ACTH has been reported mostly with ACA, whereas in our series, we observed eight patients of ACC had plasma ACTH $>20 \mathrm{pg} / \mathrm{ml}$. The varying postulates for such high ACTH values can be assay-related concerns, aberrant precursors secreted by the tumor, or intra-adrenal ACTH production.

Assay-related concerns leading to high ACTH can be attributed to the type of ACTH assay, different ACTH platforms, or heterophile antibody interference [8, 9, 29-32]. Traditionally, radioimmunoassays (RIA) were used, which also measure clinically insignificant fragments of POMC-ACTH and caused spurious elevations of ACTH. Immunoradiometric (IRMA) assays have improved this drawback as the "sandwich" complexes ensure specificity. As observed in an Italian multicentric study, plasma ACTH of $>20$ was observed in four patients of adrenal CS by RIA, as against a single case by IRMA [33]. Contemporary chemiluminescence sandwich immunoassay platforms use different capture and detection antibodies, and this can lead to a difference in ACTH measured, especially at lower ACTH levels, as shown by a study from Italy [34]. In a recent study, plasma intact ACTH level measured by LC-MS/MS highly positively correlated with plasma ACTH measured by Roche but not with that by Siemens; also, among the three discordant samples in which plasma ACTH by LC-MS/MS was undetectable, that by Roche was $<20 \mathrm{pg} / \mathrm{ml}$ in two but was more than $100 \mathrm{pg} / \mathrm{ml}$ in all when measured by Siemens [35]. As depicted in $>$ Table 3, in many cases, the ACTH detected by Siemens Immulite was much greater than Roche Elecsys performed subsequently. Similarly, all patients with ACTH of $>20 \mathrm{pg} / \mathrm{ml}$ in our series were measured on the Siemens Immulite platform. Although a repeat ACTH estimation was not available for these patients, that by Liaison assay in another five pa- tients with an initial ACTH of $13 \mathrm{pg} / \mathrm{ml}$ by Siemens Immulite assay was suppressed $(<13 \mathrm{pg} / \mathrm{ml})$ in all ( $\triangleright$ Table 3 ) suggesting a major role for assay-related issues in an apparently unsuppressed ACTH. Notably, most (8/9) of our patients with this phenomenon had ACC that makes us think of an additional role for ACC-related, yet-unidentified, factors in causing a plasma ACTH of $>20 \mathrm{pg} / \mathrm{ml}$. As conceptualized in an old study, steroidogenic precursors (21-deoxycortisol) secreted by ACC may antagonize the glucocorticoid feedback at the hypothalmic-pituitary level $[36,37]$. Although the intra-adrenal source of ACTH has been reported in PBMAH and mixed cortico-medullary adrenal tumors (MCMT), the evidence to support the production of ACTH or ACTH-like substances from ACC is negligible $[38,39]$. The evaluation for the possible role of heterophile antibodies was not performed in our study.

Hence, when ACTH is measured by the Immulite assay and is $>20 \mathrm{pg} / \mathrm{ml}$ in an endogenous CS patient, one must be cautious and rule out ACC especially in the presence of clinical or biochemical evidence of androgen excess and/or local abdominal mass effects, before ordering MRI pituitary. Repeating ACTH on a different platform using a more accurate ACTH assay and even considering adrenal imaging if clinical suspicion is high may help in correct diagnosis.

Our study is a retrospective analysis with inherent limitations. Serum DHEAS was not available in some patients. The lack of LC-MS/MS steroid profile and ACTH measurements from different platforms are other limitations. Nonetheless, all the included patients had confirmed adrenal CS with a significant sample size, and detailed clinical, biochemical and radiological features along with clinical outcomes are described.

To conclude, adrenal CS has a varied spectrum of clinical, biochemical, and imaging features that may help to differentiate malignant causes from benign ones. Cortisol-secreting ACA is usually 


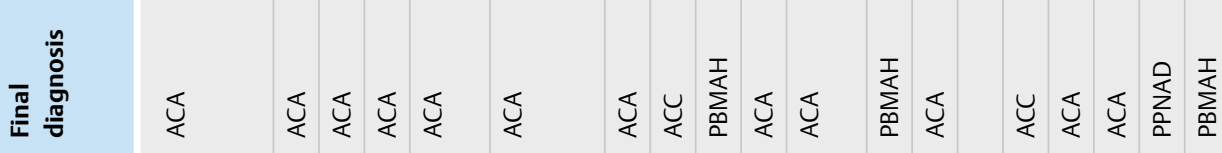

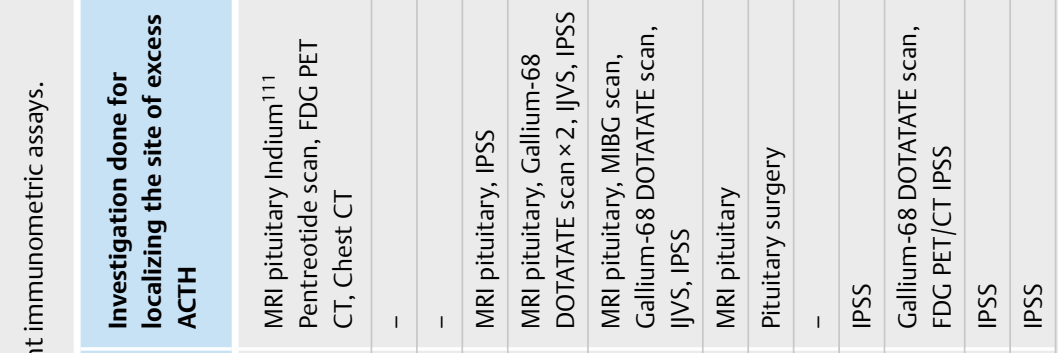

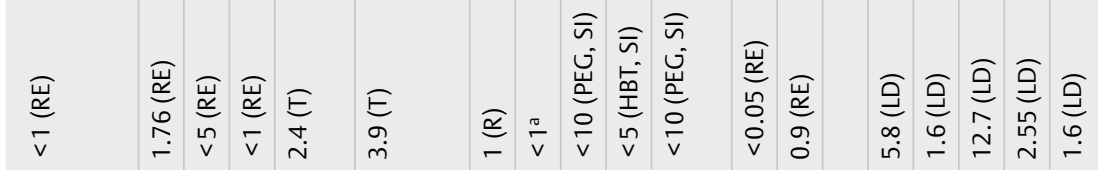

荠

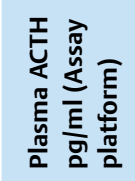

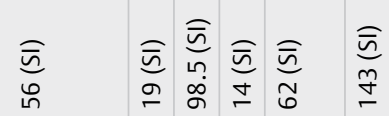

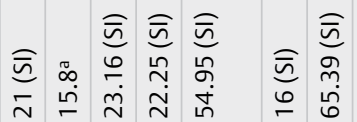

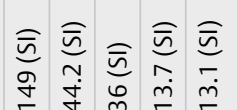

赑竞

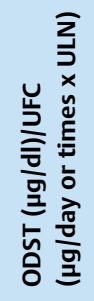

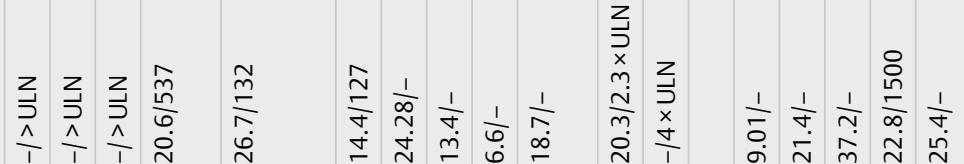

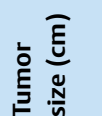

$\stackrel{\infty}{m} \underset{m}{\stackrel{\sim}{*}} \underset{m}{m} \stackrel{\sim}{\sim}$

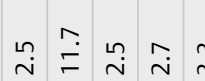

$\stackrel{m}{\sim} \stackrel{\sim}{m}$

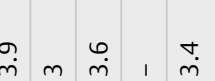

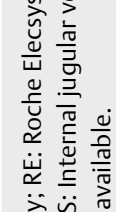

$\ddot{\alpha}$.

网覀

¿

등

㕁亮

$\stackrel{\tilde{c}}{\tilde{n}} \cdot \frac{\mathrm{c}}{\mathrm{Q}}$

离苍范

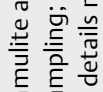

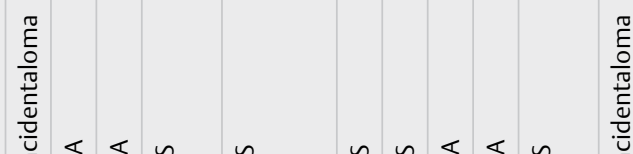

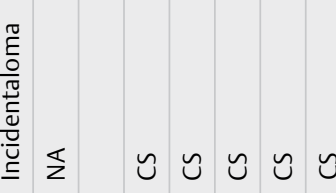

至

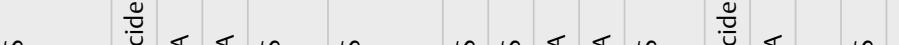

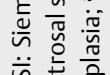

苛畜

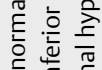

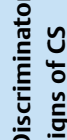

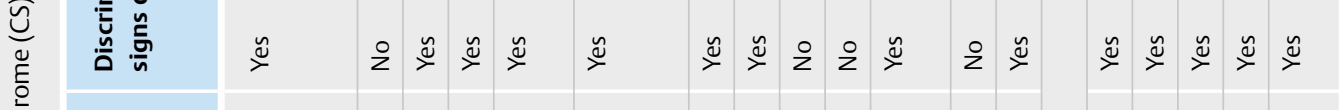

는

䓞苍

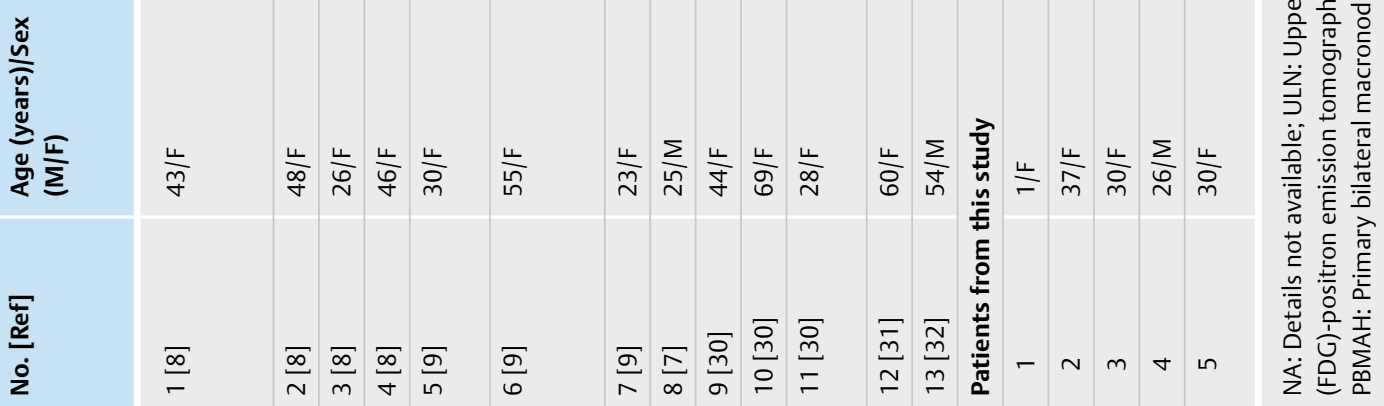


lipid-poor, and baseline $\mathrm{HU}$ is not a sensitive radiological parameter to distinguish cortisol-secreting ACA from ACC. Plasma ACTH levels may be $>20 \mathrm{pg} / \mathrm{ml}$ in adrenal CS when measured by Siemens Immulite assay, especially in patients with ACC, which can be addressed by using more accurate ACTH assays.

\section{Data Availability Statement}

The data that support the findings of this study are available on request from the corresponding author. The data is not publicly available due to privacy or ethical restrictions.

\section{Conflict of Interest}

The authors declare that they have no conflict of interest.

\section{References}

[1] Lodish M, Stratakis CA. A genetic and molecular update on adrenocortical causes of Cushing syndrome. Nat Rev Endocrinol 2016; 12: $255-262$

[2] Stratakis CA. Cushing syndrome caused by adrenocortical tumors and hyperplasias (corticotropin-independent Cushing syndrome). Endocr Dev 2008; 13: 117-132

[3] Else T, Kim AC, Sabolch A et al. Adrenocortical carcinoma. Endocr Rev 2014; 35: 282-326

[4] Albiger NM, Ceccato F, Zilio M et al. An analysis of different therapeutic options in patients with Cushing's syndrome due to bilateral macronodular adrenal hyperplasia: a single-centre experience. Clin Endocrinol (Oxf) 2015; 82: 808-815

[5] Sharma ST. AACE Adrenal Scientific Committee. An individualized approach to the evaluation of Cushing syndrome. Endocr Pract 2017; 23: 726-737

[6] Lacroix A, Feelders RA, Stratakis CA et al. Cushing's syndrome. Lancet 2015; 386: 913-927

[7] Jarial KD, Walia R, Kumar S et al. Adrenocortical carcinoma masquerading as Cushing's disease. BMJ Case Rep 2017; bcr2016217519

[8] Donegan DM, Algeciras-Schimnich A, Hamidi O et al. Corticotropin hormone assay interference: A case series. Clin Biochem 2019; 63: 143-147

[9] Greene LW, Geer EB, Page-Wilson G et al. Assay-specific spurious ACTH results lead to misdiagnosis, unnecessary testing, and surgical misadventure - a case series. J Endocr Soc 2019; 3: 763-772

[10] Valassi E, Franz H, Brue T et al. Diagnostic tests for Cushing's syndrome differ from published guidelines: data from ERCUSYN. Eur J Endocrinol 2017; 176: 613-624

[11] Nieman LK, Biller BMK, Findling JW et al. The diagnosis of Cushing's syndrome: an endocrine society clinical practice guideline. J Clin Endocrinol Metab 2008; 93: 1526-1540

[12] Goroshi M, Jadhav SS, Sarathi V et al. Radiological differentiation of phaeochromocytoma from other malignant adrenal masses: importance of wash-in characteristics on multiphase CECT. Endocr Connect 2019; 8: 898-905

[13] Elmlinger MW, Kühnel W, Ranke MB. Reference ranges for serum concentrations of lutropin (LH), follitropin (FSH), estradiol (E2), prolactin, progesterone, sex hormone-binding globulin (SHBG), dehydroepiandrosterone sulfate (DHEAS), cortisol and ferritin in neonates, children and young adults. Clin Chem Lab Med 2002; 40: 1151-1160. doi:10.1515/CCLM.2002.202

[14] Nakamoto J. Endocrine testing. In: Endocrinology: Adult and Pediatric. Philadelphia: Elsevier Saunders; 2016

[15] Memon SS, Thakkar K, Patil V et al. Primary pigmented nodular adrenocortical disease (PPNAD): single centre experience. J Pediatr Endocrinol Metab 2019; 32: 391-397

[16] Ammini AC, Tandon N, Gupta N et al. Etiology and clinical profile of patients with Cushing's syndrome: a single center experience. Indian J Endocrinol Metab 2014; 18: 99-105

[17] Tella SH, Kommalapati A, Yaturu S et al. Predictors of survival in adrenocortical carcinoma: an analysis from the national cancer database. J Clin Endocrinol Metab 2018; 103: 3566-3573

[18] Ayala-Ramirez M, Jasim S, Feng L et al. Adrenocortical carcinoma: clinical outcomes and prognosis of 330 patients at a tertiary care center. Eur J Endocrinol 2013; 169: 891-899

[19] Danilowicz K, Albiger N, Vanegas M et al. Androgen-secreting adrenal adenomas. Obstet Gynecol 2002; 100: 1099-1102

[20] Chentli F, Bekkaye I, Azzoug S. Feminizing adrenocortical tumors: literature review. Indian J Endocrinol Metab 2015; 19: 332-339

[21] Di Dalmazi G, Berr CM, Fassnacht M et al. Adrenal function after adrenalectomy for subclinical hypercortisolism and Cushing's syndrome: a systematic review of the literature. J Clin Endocrinol Metab 2014; 99: 2637-2645

[22] Boland GW, Lee M], Gazelle GS et al. Characterization of adrenal masses using unenhanced CT: an analysis of the CT literature. AJR Am J Roentgenol 1998; 171: 201-204

[23] Humbert A-L, Lecoanet G, Moog S et al. The computed tomography adrenal wash-out analysis properly classifies cortisol secreting adrenocortical adenomas. Endocrine 2018; 59: 529-537

[24] Chambre C, McMurray E, Baudry C et al. The 10 Hounsfield units unenhanced computed tomography attenuation threshold does not apply to cortisol secreting adrenocortical adenomas. Eur J Endocrinol 2015; 173: 325-332

[25] Jia A-H, Du H-Q, Fan M-H et al. Clinical and pathological analysis of 116 cases of adult adrenal cortical adenoma and literature review. Onco Targets Ther 2015; 8: 1251-1257

[26] Petersenn S, Richter P-A, Broemel T et al. Computed tomography criteria for discrimination of adrenal adenomas and adrenocortical carcinomas: analysis of the German ACC registry. Eur J Endocrinol 2015; 172: 415-422

[27] Sangwaiya M], Boland GWL, Cronin CG et al. Incidental adrenal lesions: accuracy of characterization with contrast-enhanced washout multidetector CT-10-minute delayed imaging protocol revisited in a large patient cohort. Radiology 2010; 256: 504-510

[28] He X, Caoili EM, Avram AM et al. 18F-FDG-PET/CT Evaluation of indeterminate adrenal masses in noncancer patients. J Clin Endocrinol Metab 2021; 106: 1448-1459

[29] Bolstad N, Warren DJ, Nustad K. Heterophilic antibody interference in immunometric assays. Best Pract Res Clin Endocrinol Metab 2013; 27: 647-661

[30] Yener S, Demir L, Demirpence M et al. Interference in ACTH immunoassay negatively impacts the management of subclinical hypercortisolism. Endocrine 2017; 56: 308-316

[31] Grasko J, Williams R, Beilin J et al. A diagnostic conundrum: heterophilic antibody interference in an adrenocorticotropic hormone immunoassay not detectable using a proprietary heterophile blocking reagent. Ann Clin Biochem 2013; 50: 433-437

[32] Morton A, Dover T. Heterophile antibody to adrenocorticotropin hormone interfering with the investigation of Cushing's syndrome. Indian J Clin Biochem 2019; 34: 234-236 
[33] Invitti C, Pecori Giraldi F, de Martin M et al. Diagnosis and management of Cushing's syndrome: results of an Italian multicentre study. Study group of the Italian society of endocrinology on the pathophysiology of the hypothalamic-pituitary-adrenal axis. J Clin Endocrinol Metab 1999; 84: 440-448

[34] Pecori Giraldi F, Saccani A, Cavagnini F et al. Assessment of ACTH assay variability: a multicenter study. Eur J Endocrinol 2011; 164: 505-512

[35] Shi J, Dhaliwal P, Zi Zheng Y et al. An intact ACTH LC-MS/MS assay as an arbiter of clinically discordant immunoassay results. Clin Chem 2019; 65: 1397-1404
[36] Taylor DR, Ghataore L, Couchman L et al. A 13-tseroid serum panel based on LC-MS/MS: use in detection of adrenocortical carcinoma. Clin Chem 2017; 63: 1836-1846

[37] Ismail AA, Burr WA, Taylor NF et al. Elevated plasma adrenocorticotropin (ACTH) with adrenal hyperplasia: a new factor in ACTH regulation? J Clin Endocrinol Metab 1991; 73: 752-757

[38] Louiset E, Duparc C, Young J et al. Intraadrenal corticotropin in bilateral macronodular adrenal hyperplasia. N Engl J Med 2013; 369: 2115-2125

[39] Lwin TM, Galal N, Gera S et al. Adrenal Cushing syndrome with detectable ACTH from an unexpected source. BMJ Case Rep 2016; 2016: bcr2016216965 